\title{
A re-evaluation of the effects of dual growing rods on apical vertebral rotation in patients with early-onset scoliosis and a minimum of two lengthening procedures: a CT-based study
}

\author{
Liang Xu, MD, Yong Qiu, MD, Zhonghui Chen, MD, Benlong Shi, MD, Xi Chen, MD, Song Li, MD, \\ Changzhi Du, MD, Zezhang Zhu, MD, and Xu Sun, MD
}

Department of Spine Surgery, Drum Tower Hospital, Nanjing University Medical School, Nanjing, Jiangsu Province, China

OBJECTIVE This study aimed to evaluate the correction results of traditional dual growing rods (DGRs) on axial rotation using $\mathrm{CT}$ scans and to further explore the relationships between axial and torso deformities in patients with earlyonset scoliosis (EOS).

METHODS Patients with EOS who were treated with traditional DGRs between January 2006 and December 2014 were retrospectively reviewed. Plain radiographs were used to assess the degree of coronal and sagittal deformity. The apical vertebral rotation (AVR) and rib hump (RH) were measured on CT scans at the apical vertebra. Pearson or Spearman rank correlation analyses were used to analyze the associations between spinal and torso deformities.

RESULTS A total of 27 patients ( 10 boys and 17 girls, average age $6.5 \pm 1.7$ years) were enrolled in this study. The average number of lengthenings per patient was $5.0 \pm 1.9$, with a mean follow-up duration of $52.9 \pm 18.2$ months. The apical vertebral translation, apical vertebral body-rib ratio (AVB-R), AVR, and RH parameters were significantly decreased after the initial surgery $(p<0.05)$ but showed notable progression at the latest follow-up evaluation $(p<0.05)$. The preoperative AVR and its correction after index surgery were significantly correlated with the preoperative values as well as with the corrections of the major Cobb angle, AVB-R, and RH. During the follow-up period, significant correlations were found between the deterioration of AVR and the AVB-R and also between the deterioration of AVR and the RH from the initial surgery to the latest follow-up.

CONCLUSIONS Significant AVR correction can be achieved by DGR techniques after the initial surgery. However, this technique weakly prevents the deterioration of AVR during the follow-up period.

https://thejns.org/doi/abs/10.3171/2018.3.PEDS1832

KEYWORDS apical vertebral rotation; growing rods; early-onset scoliosis; spine

$\mathrm{E}$ ARLY-ONSET scoliosis (EOS) refers to a complex 3D and rapidly progressed deformity. $4,15,23$ Through periodical distraction across the nonfused portion of the deformed spine, the traditional dual growing rod (DGR) technique has been widely used in the treatment of patients with EOS due to its effectiveness in controlling coronal and sagittal deformity while also permitting pulmonary system development., ${ }^{9,13,16}$ However, because this growth-friendly technique simply combines upper and lower instrumentation with dual rods, its limitation of lacking apical vertebral control has remained a concern.

Apical vertebral rotation (AVR) on the axial plane is an integral part of deformity, contributing to both coronal and cosmetic deformities as well as to respiratory dysfunction during spinal deformity progression. ${ }^{14,27}$ In patients with EOS, failure to control the AVR during lengthening procedures may substantially influence the correction results at the final fusion, potentially leading to unsatisfactory trunk

ABBREVIATIONS AVB-R = apical vertebral body-rib ratio; AVR = apical vertebral rotation; $A V T$ = apical vertebral translation; $D G R=$ dual growing rod; EOS = early-onset scoliosis; ICC = intraclass correlation coefficient; $\mathrm{LL}=$ lumbar lordosis; $\mathrm{RH}=$ rib hump; TK = thoracic kyphosis.

SUBMITTED January 12, 2018. ACCEPTED March 14, 2018.

INCLUDE WHEN CITING Published online June 15, 2018; DOI: 10.3171/2018.3.PEDS1832. 
cosmesis with rib hump (RH), limited scoliosis correction, restricted pulmonary volume gain, and the unexpected crankshaft phenomenon. ${ }^{11}$ Thus, accurately quantifying the AVR in patients with EOS may be vitally important for evaluating the effect of growing rods. Among different radiological evaluation methods, $\mathrm{CT}$ is regarded as the most accurate because of its advantages in identifying key anatomical bone landmarks on the axial plane. ${ }^{19,20}$ However, axial deformity analyses with CT have rarely been reported in patients with EOS.

In a previous study, Kamaci et al. ${ }^{17}$ reported the effectiveness of traditional DGRs in controlling axial rotation after analyzing 12 patients with EOS who underwent CT scans at their latest follow-up. However, this study's limitations of a small sample size and lack of CT scanning before and after the initial surgery potentially made the effects of DGRs on axial deformity correction inconclusive. In addition, research on the relationship between AVR and torso deformity is still lacking. Thus, the present study aimed to: 1) evaluate the correction results of traditional DGRs on axial rotation by retrospectively analyzing patients with EOS who underwent CT scans both before and after the initial surgery as well as during their latest follow-up; and 2) further explore the relationships between axial rotation and torso deformity in patients with EOS.

\section{Methods \\ Study Sample}

After approval from the IRB, a retrospective review of a single database comprising patients with EOS from January 2006 to December 2014 was performed. The patient enrollment criteria were as follows: 1) treated with traditional DGRs; 2) had at least 2 lengthening procedures together with 2 years of follow-up after the initial surgery; and 3) underwent both radiographic and CT scanning before surgery, immediately after surgery, and at the latest follow-up. The patient exclusion criteria were as follows: 1) prior history of spinal surgery and halo gravity traction before the index surgery; and 2) hemivertebrae at the apical level.

CT scans were performed in the supine position preoperatively to provide information on the severity of the deformed bony vertebral structure to guide the selection and insertion of appropriate pedicle screws, and postoperatively to detect any malpositioned pedicle screws potentially causing neural or vascular injury or impingement. During the follow-up period, CT scans were performed only on patients with suspicious implant dislodgement and/or notching, regardless of the AVR severity on radiographs. All CT scans were performed with a spiral CT scanner (Lightspeed 16, GE Medical Systems) using the following protocol: $120 \mathrm{kV}$; tube current $100-350 \mathrm{~mA}$; gantry rotation 0.8 seconds; feed $27.5 \mathrm{~mm} /$ rotation; and slice thickness and detector collimation of 1.25 and $5 \mathrm{~mm}$, respectively.

The following patient demographics were collected: diagnosis, age at index surgery, and number and intervals of the lengthening procedures. For patients with definitive fusion, the latest follow-up referred to the time point prior to the definitive fusion procedure.

\section{Surgical Technique}

During the index surgery, implants were placed with small, separate incisions, and only cephalad and caudal anchor sites were exposed subperiosteally. Both proximal and distal fixations were placed over 2 levels in all cases. Generally, the proximal and distal foundation levels were chosen from $\mathrm{T} 2$ to $\mathrm{T} 5$ and from L2 to L5, respectively. Pedicle screws were preferably placed at anchoring sites, but hooks were used otherwise. The vertebral segments between the anchors remained undissected to prevent autofusion. Next, the 4 overlapping rods were tunneled with 2 rod-to-rod connectors in a submuscular manner to reduce soft-tissue trauma and infection. During the followup period, the interval between subsequent lengthening procedures was scheduled for approximately $8-10$ months. Somatosensory-evoked potentials and motor-evoked potentials were recorded for all surgical procedures.

\section{Radiographic Evaluation}

The vertebra of the curve most laterally deviated from the central sacral vertical line was chosen as the apical vertebra. Standing posteroanterior radiographs were used for measurements of the following parameters: 1) major coronal Cobb angle; 2) T1-S1 height, defined as the vertical distance between the middle points of the superior endplates of T1 and S1; 3) thoracic kyphosis (TK), the angle between the superior endplate of T2 and the inferior endplate of T12; 4) lumbar lordosis (LL), the angle between the superior endplate of L1 and the superior endplate of S1; 5) apical vertebral translation (AVT), the distance from the central sacral vertical line to the midpoint of the apical vertebra; and 6) the apical vertebral body-rib ratio (AVB-R), the ratio of linear measurements from the lateral borders of the apical thoracic vertebrae to the chest wall.

Parameters at the apical level measured by CT (Fig. 1) were as follows. First, the AVR was measured, ${ }^{1,2}$ the angle between two longitudinal axes of the apical vertebra and the pelvis. The former line was drawn from the most posterior corner of the spinal canal extending anteriorly to equally bisect the vertebral body. The latter line was defined as the line vertical to the anterior margins of the left and right sacroiliac joints at the CT slice that passed through the first sacral vertebra. Second, the RH was measured, the linear distance between the left and right posterior rib prominences at the apex of the rib deformity.

In this study, all radiographs were measured twice at an interval of 1 week by a well-trained senior resident, and the mean of both measurements was adopted for analysis in this study. The intraobserver reproducibility of all measurements obtained was tested and quantified by the intraclass correlation coefficient (ICC). There were strong intraobserver agreements for all parameters, as all ICCs exceeded 0.8 .

\section{Statistical Analysis}

Statistical analysis was performed using the IBM SPSS Statistics software package. The paired t-test was used for intragroup comparisons among pre- and postoperative variables as well as at the latest follow-up. Correlations 


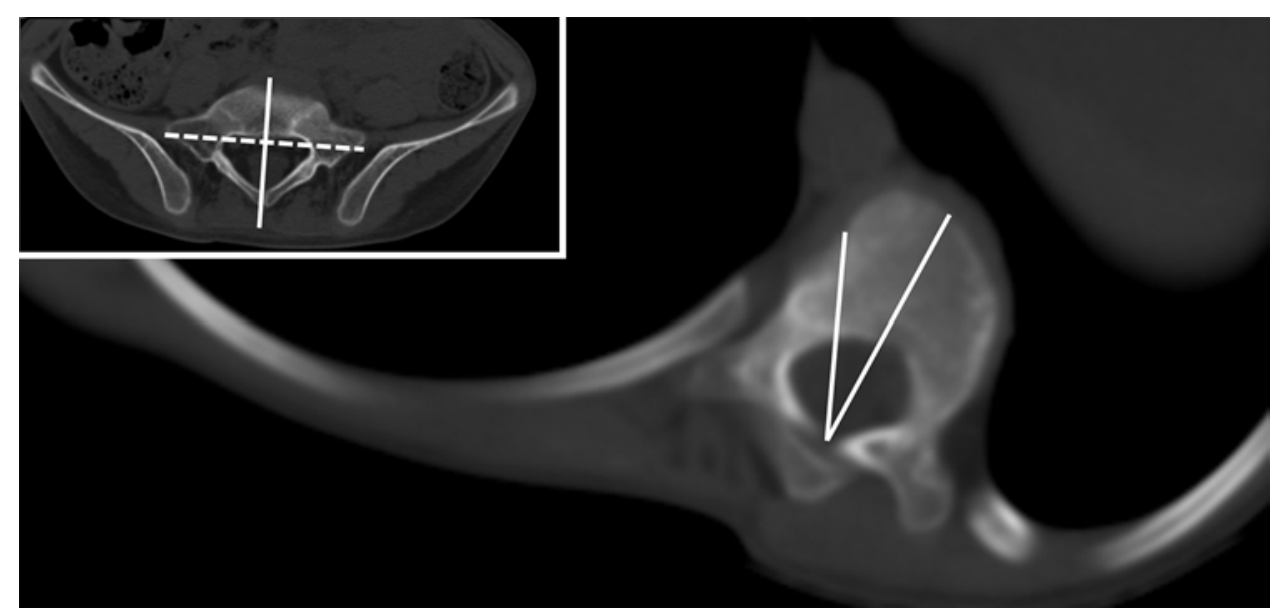

FIG. 1. Illustration of the AVR, the angle between two longitudinal axes of the apical vertebra and the pelvis.

between parameters were evaluated with Pearson correlation analysis for normally distributed parameters and with Spearman rank correlation analysis for nonnormally distributed parameters; $\mathrm{p}$ values $<0.05$ were considered statistically significant.

\section{Results \\ Demographics}

Of the 78 patients treated with traditional DGRs in our database, 27 were enrolled in this study (average age 6.5 \pm 1.7 years, 10 boys and 17 girls). Among them, the etiologies were classified as idiopathic in 6 patients, syndromic in 2 , neuromuscular in 6 , and congenital in 13 . Fourteen hooks were placed as anchors proximally in 4 patients (12 hooks) and distally in 1 patient (2 hooks). A total of 137 lengthening procedures were performed, with an average of $5.0 \pm 1.9$ procedures performed per patient. The mean lengthening interval and follow-up duration were $10.6 \pm$ 0.9 months and $52.9 \pm 18.2$ months, respectively. Two patients who reached skeletal maturity underwent removal of the DGR instrumentation and definitive fusion.

\section{General Correction Results}

As shown in Table 1, the major Cobb angle was sig- nificantly improved after the initial surgery $\left(64.9^{\circ} \pm 8.3^{\circ}\right.$ vs $\left.32.9^{\circ} \pm 6.1^{\circ}, \mathrm{p}<0.001\right)$ and remained steady over subsequent distractions $(\mathrm{p}=0.094)$. After the index surgery, T1-S1 had an average annual height gain of $1.1 \pm 0.3 \mathrm{~cm}$ through the latest follow-up. On the sagittal plane, both TK and LL were remarkably decreased after the initial surgery and slightly increased throughout the follow-up period. Through the latest follow-up evaluation, a total of 14 complications occurred in 12 patients, including proximal junctional kyphosis in 5 patients, rod fractures in 4 patients, screw pullout in 2 patients, superficial infections in 2 patients, and dislodgement in 1 patient. No unplanned surgical procedures were required. Patients with proximal junctional kyphosis were followed by observation or bracing, and none developed proximal junctional failure during follow-up. Patients with superficial infection were successfully managed by dressing changing, and those with rod fractures were treated with a single unplanned surgery.

\section{Evaluations of AVR and Torso Deformity}

On CT scans, the mean AVR was significantly improved from $28.1^{\circ} \pm 8.0^{\circ}$ preoperatively to $21.5^{\circ} \pm 5.6^{\circ}$ postoperatively, with a mean correction rate of $21.2 \% \pm$

TABLE 1. Results of the general correction, spinal growth, axial rotation, and torso deformity

\begin{tabular}{lrrrrrrr}
\hline & & & & Preop to & Postop to & \multicolumn{2}{c}{$p$ Value } \\
\cline { 7 - 8 } \multicolumn{1}{c}{ Measurement } & Preop & Postop & Latest FU & Postop & Latest FU & Preop vs Postop & Postop vs Latest FU \\
\hline Major Cobb angle $\left(^{\circ}\right)$ & $64.9 \pm 8.3$ & $32.9 \pm 6.1$ & $34.5 \pm 7.2$ & $32.0 \pm 7.3$ & $-1.6 \pm 4.8$ & $<0.001$ & 0.094 \\
\hline T1-S1 height $(\mathrm{cm})$ & $26.6 \pm 2.7$ & $29.2 \pm 2.6$ & $34.1 \pm 3.3$ & $2.6 \pm 0.4$ & $-4.9 \pm 1.6$ & $<0.001$ & $<0.001$ \\
\hline TK $\left(^{\circ}\right)$ & $53.1 \pm 6.0$ & $38.5 \pm 4.8$ & $40.4 \pm 7.8$ & $14.5 \pm 7.0$ & $-1.8 \pm 5.7$ & 0.021 & 0.114 \\
\hline $\mathrm{LL}\left({ }^{\circ}\right)$ & $50.6 \pm 4.9$ & $42.4 \pm 4.6$ & $44.9 \pm 7.8$ & $8.1 \pm 6.2$ & $-2.4 \pm 8.1$ & $<0.001$ & 0.130 \\
\hline $\mathrm{AVT}(\mathrm{mm})$ & $34.2 \pm 7.6$ & $26.1 \pm 6.1$ & $28.7 \pm 6.5$ & $8.1 \pm 9.4$ & $-2.6 \pm 6.0$ & $<0.001$ & 0.031 \\
\hline AVB-R & $2.1 \pm 0.4$ & $1.7 \pm 0.4$ & $1.9 \pm 0.5$ & $0.4 \pm 0.4$ & $-0.2 \pm 0.3$ & $<0.001$ & 0.003 \\
\hline AVR $\left({ }^{\circ}\right)$ & $28.1 \pm 8.0$ & $21.5 \pm 5.6$ & $25.8 \pm 7.7$ & $6.5 \pm 6.0$ & $-4.3 \pm 5.7$ & $<0.001$ & 0.001 \\
\hline $\mathrm{RH}(\mathrm{mm})$ & $21.7 \pm 5.3$ & $13.1 \pm 5.2$ & $18.6 \pm 6.2$ & $8.5 \pm 5.6$ & $-5.4 \pm 6.2$ & $<0.001$ & $<0.001$ \\
\hline
\end{tabular}

$\mathrm{FU}=$ follow-up.

Means are presented \pm standard deviations. 

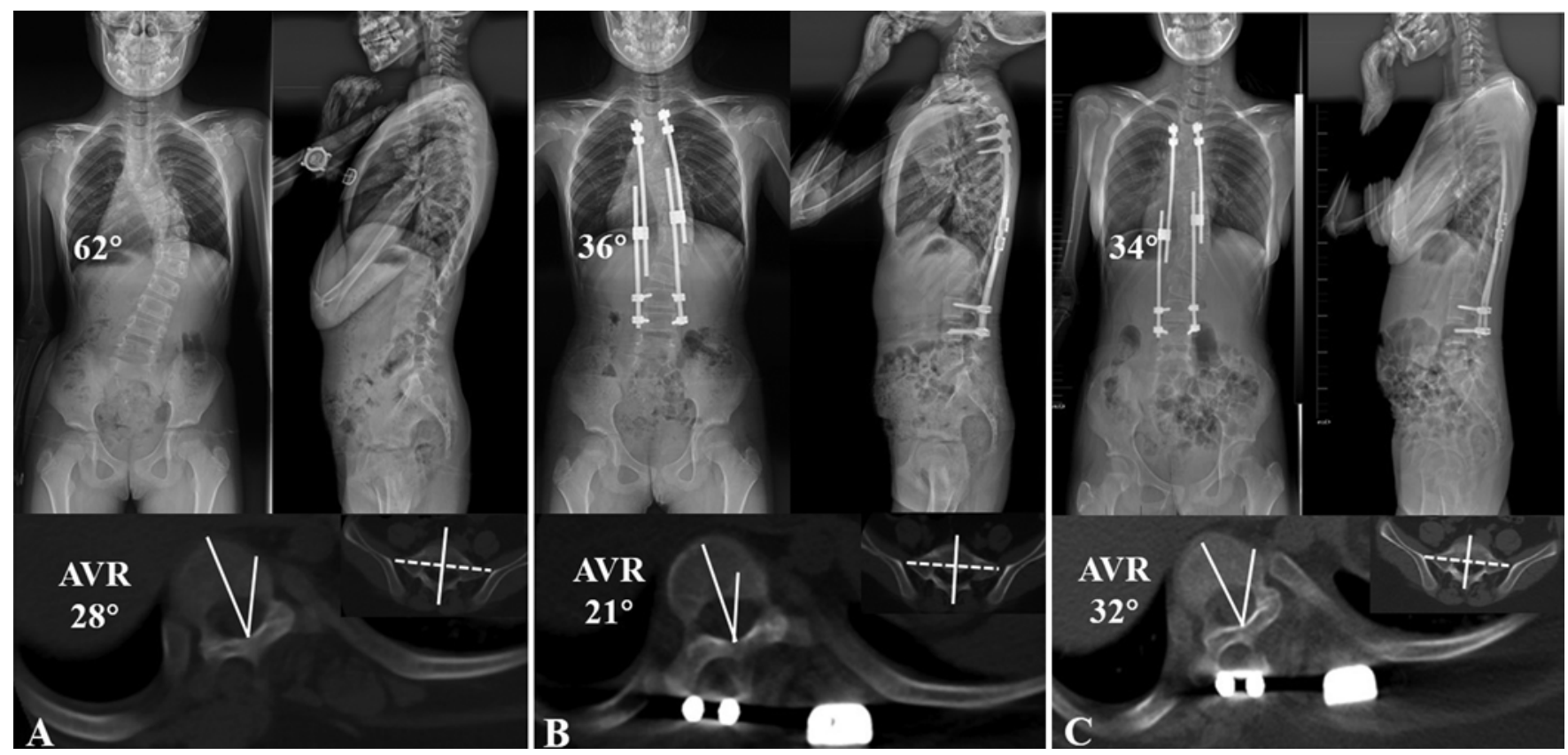

FIG. 2. A: Standing radiographs (upper) and axial CT images (lower) from a 7-year-old girl with congenital EOS who received treatment using DGRs. B: Both the main curve and AVR were remarkably corrected postoperatively. C: After 51 months of followup (5 lengthenings), the main curve was maintained whereas the AVR significantly increased to $32^{\circ}$.

$16.9 \%(\mathrm{p}<0.001)$. At the latest follow-up, the mean AVR was $25.8^{\circ} \pm 7.7^{\circ}$, with a mean correction loss of $4.3^{\circ} \pm$ $5.7^{\circ}$. In addition, statistical analysis demonstrated that the AVR changed significantly with repeated lengthening ( $\mathrm{p}$ $=0.001)$. Similarly, the RH was significantly corrected from $21.7 \pm 5.3 \mathrm{~mm}$ to $13.1 \pm 5.2 \mathrm{~mm}$. The preoperative AVB-R and AVT averaged $2.1 \pm 0.4$ and $34.2 \pm 7.6 \mathrm{~mm}$, respectively, and both values were significantly corrected after the index surgery. However, notable aggravations of the above parameters were found at the latest follow-up (Fig. 2).
For the 2 patients with definitive fusion, although the major Cobb angle was notably improved (from $40^{\circ}$ to $32^{\circ}$ for patient 1 [Fig. 3] and from $48^{\circ}$ to $39^{\circ}$ for patient 2), changes in the AVR after surgery were not significant (from $29^{\circ}$ to $25^{\circ}$ for patient 1 and from $35^{\circ}$ to $32^{\circ}$ for patient 2).

\section{Correlation Among Spinal Parameters}

As shown in Table 2, the preoperative AVR and its correction after index surgery were significantly correlated with the respective preoperative values as well as with the
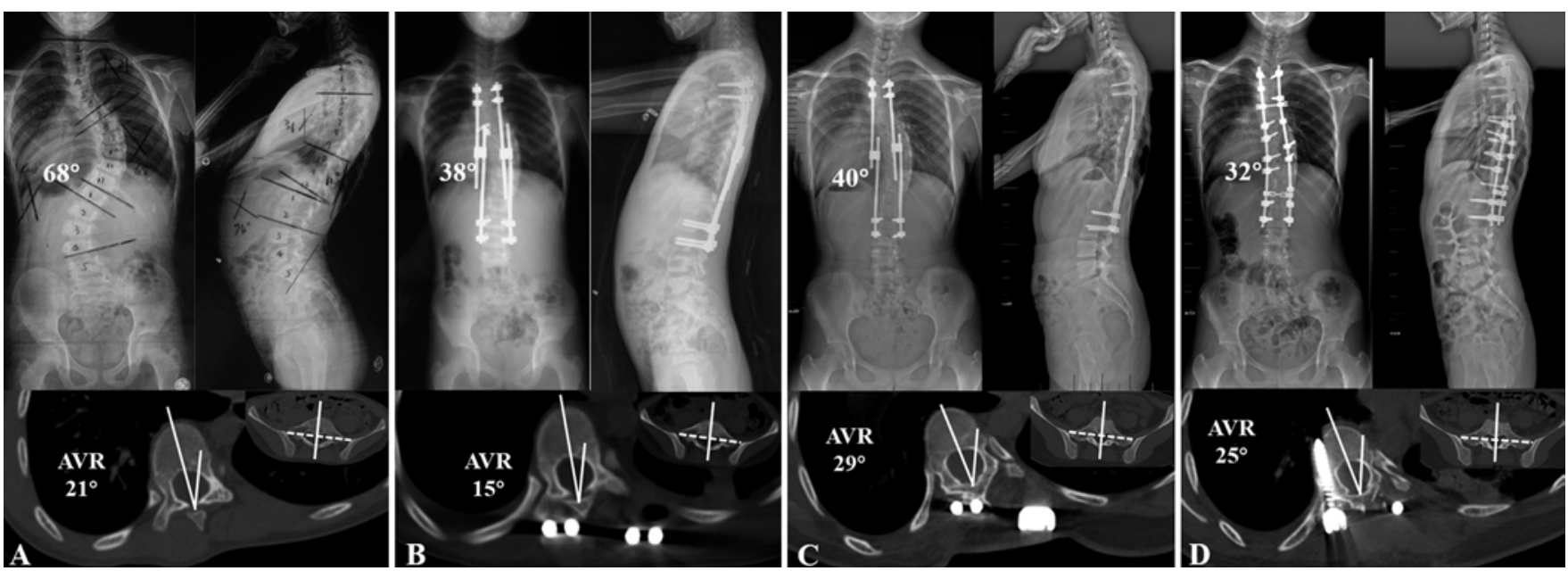

FIG. 3. A: Standing radiographs (upper) and axial CT images (lower) from an 8-year-old girl with idiopathic EOS who received surgical treatment with DGRs. B: After the initial surgery, the AVR was remarkably corrected. C: After completion of the 7-year follow-up period (6 lengthenings), definitive fusion was performed, but the AVR notably increased. D: Although definitive fusion was performed, nonsignificant corrections in the AVR were noted. 
TABLE 2. Correlation analysis between AVR and spinal as well as torso deformity

\begin{tabular}{|c|c|c|c|c|c|c|c|c|c|c|}
\hline \multirow[b]{2}{*}{ Variable } & \multicolumn{2}{|c|}{ Preop } & \multicolumn{2}{|c|}{ Postop } & \multicolumn{2}{|c|}{ Latest FU } & \multicolumn{2}{|c|}{ Preop to Postop } & \multicolumn{2}{|c|}{ Postop to Latest FU } \\
\hline & rValue & $p$ Value & r Value & $p$ Value & r Value & p Value & rValue & p Value & r Value & $p$ Value \\
\hline Major Cobb angle & 0.512 & 0.006 & 0.134 & 0.506 & 0.367 & 0.060 & 0.443 & 0.021 & -0.023 & 0.911 \\
\hline TK & -0.097 & 0.629 & 0.053 & 0.794 & -0.189 & 0.345 & -0.135 & -0.503 & 0.037 & 0.854 \\
\hline LL & 0.082 & 0.686 & 0.316 & 0.108 & -0.049 & 0.807 & 0.095 & 0.639 & -0.073 & 0.718 \\
\hline AVT & -0.079 & 0.694 & -0.153 & 0.445 & -0.120 & 0.551 & 0.167 & 0.406 & -0.029 & 0.884 \\
\hline AVB-R & 0.539 & 0.004 & 0.252 & 0.205 & 0.591 & 0.001 & 0.447 & 0.019 & 0.509 & 0.007 \\
\hline $\mathrm{RH}$ & 0.556 & 0.003 & 0.108 & 0.593 & 0.346 & 0.077 & 0.605 & 0.001 & 0.640 & $<0.001$ \\
\hline
\end{tabular}

$\mathrm{r}$ value $=$ correlation coefficient .

corrections of the major Cobb angle, AVB-R, and RH. Regarding the changes occurring between the postoperation period and the latest follow-up, significant correlations were found only between the deterioration of AVR and $\mathrm{RH}$ and also between the deterioration of AVR and AVBR. Figure 2 shows the radiographic and CT evaluations of a spinal deformity case under DGR treatment with a 51-month follow-up.

\section{Discussion}

The current study demonstrated that DGRs satisfactorily corrected coronal deformities after index surgery and maintained steady results through the latest follow-up evaluation in a cohort of 27 patients. Simultaneously, the annual T1-S1 gain in our cohort (1.1 cm/year) was comparable to those in previous reports (ranging from 1.01 to $1.84 \mathrm{~cm} /$ year). ${ }^{5,6,26}$ Regarding axial deformity, notable AVR correction was achieved after the initial surgery, but significant deterioration was also noted over a mean follow-up period of $52.9 \pm 18.2$ months. Based on these data, traditional DGRs were effective in correcting axial rotation after index surgery but was unable to prevent AVR deterioration during the follow-up period.

The previously established effects of growth-friendly techniques on axial deformity correction remain controversial. Kamaci et al. ${ }^{17}$ reported that DGRs effectively corrected axial deformity by analyzing 12 patients with EOS who underwent CT scans at their latest follow-up evaluation. On the other hand, inaccurate measurements of axial deformity by radiography eventually influence the axial rotation evaluation. Moreover, the relatively small sample size of 12 patients also made the effects of DGRs on vertebral rotation inconclusive. In contrast, several reports have noted poor effects on axial deformity correction during the follow-up period. ${ }^{3,11,24}$ Enercan et al. ${ }^{11}$ reported that lacking apical control during follow-up was an inevitable limitation of treatment with the DGR technique. By accurately evaluating axial deformities on CT scans before and after surgery as well as during the follow-up period, similar poor results in AVR control during the follow-up period were also observed in the current study. Due to both the autofusion phenomenon and rigid spinal deformity at the apical level, poor AVR correction outcomes were also noted in 2 patients in the current study even when definitive fusion was performed (Fig. 3).

Our study also demonstrated notable torso deformity progression in these patients with EOS during follow-up. In agreement with the results of Little et al., ${ }^{20}$ who reported that the change in AVR was significantly correlated with changes in the major Cobb angle and the RH, significant correlations between the deterioration of AVR and RH and also between the deterioration of AVR and AVB-R were noted in the current study. Together, these results demonstrate that more AVR progression is definitively associated with the deterioration of torso deformity, which consequently aggravates the unsatisfactory cosmesis. Moreover, based on the relationship between torso deformity and pulmonary insufficiency established in previous studies,, 28 we hypothesized that the deterioration of AVR during the follow-up period may negatively influence pulmonary insufficiency.

In contrast to the notable correlation between AVR progression and aggravated torso deformity during follow-up, no significant correlations were observed between the deterioration of AVR and the change in the major Cobb angle from the postoperative period to the latest follow-up. This result may have been due to the existence of a more complicated relationship between axial and coronal deformity during the follow-up period, as surgical distraction in the form of DGRs during each lengthening procedure mainly corrected the frontal plane component and failed to control and derotate the vertebral rotation in the axial plane, leaving an unparalleled change between the axial rotation and the major Cobb angle. In addition, this unparalleled pattern may eventually lead to the crankshaft phenomenon as well as to the diminished returns on deformity correction during future lengthening procedures. ${ }^{10,22}$

The precise mechanism underlying the deterioration of axial rotation in patients treated with DGRs remains unknown. Young patients less than 10 years of age have been regarded as an independent risk factor for the deterioration of AVR in previous studies..$^{18,25}$ Moreover, asymmetrical growth of the apical vertebral pedicle also contributes to the progressive vertebral rotation in EOS.,30 Because this growth-friendly DGR technique simply combines upper and lower instrumentation with dual rods, its limitations of lacking apical control and interfering with asymmetrical growth potential in the apical vertebra may be the main causes of AVR deterioration during the follow-up period. ${ }^{3,11,24}$ In addition to the lack of apical control, the autofusion phenomenon has also been reported as a common complication during follow-up. Unexpected posterior 
tethering caused by autofusion combined with continued growth in the anterior column could also eventually lead to both increased axial rotation and the crankshaft phenomenon..$^{8,12}$

To the best of our knowledge, few studies exist on strategies for enhancing AVR correction using growth-friendly techniques. In a retrospective study on patients with congenital scoliosis, Wang et al. ${ }^{29}$ reported a hybrid growing rod technique using hemivertebrectomy and short fusion to strengthen apical control. In addition, the Shilla growth guidance system, which maximally corrects the spinal deformity apex while guiding cephalad and caudal spinal growth along spinal rods, was also recently reported. ${ }^{21}$ However, whether these modified growth-friendly techniques eventually prevent the progression of AVR during follow-up needs further investigation.

Several limitations of this study must be addressed. First, the strength of the results was limited by the relatively small sample size as well as by the short follow-up duration. In addition, only 2 patients in the current study underwent definitive surgery. A long-term follow-up study is required for an in-depth evaluation of the correlations between higher AVR and definitive surgical outcomes. Second, the evaluations of correlations among greater AVR, pulmonary insufficiency, and health-related quality of life were limited due to the retrospective nature of the current study. Finally, the etiological variety of our cohort may have created inherent drawbacks in making generalized statements. Despite these limitations, the current study accurately evaluated axial deformity on CT scans and noted that the DGR technique was weak in controlling vertebral rotation during lengthening, indicating that this technique needs to be modified in the future.

\section{Conclusions}

Treatment with DGRs is effective in correcting scoliosis while allowing spinal growth. In the axial plane, DGRs weakly prevented the deterioration of AVR during the follow-up period despite significant AVR correction after the index surgery with DGR implant placement. Moreover, the progression of AVR during the follow-up period was significantly correlated with the change in torso deformity. AVR deterioration might be correlated with the substantial growth potential, lack of apical control, and intravertebral asymmetrical growth in the axial plane.

\section{Acknowledgments}

This study was supported by the National Natural Science Foundation of China (Grant No. 81772422) and the Natural Science Foundation of Jiangsu Province (BK20170126).

\section{References}

1. Aaro S, Dahlborn M: Estimation of vertebral rotation and the spinal and rib cage deformity in scoliosis by computer tomography. Spine (Phila Pa 1976) 6:460-467, 1981

2. Aaro S, Dahlborn M: The longitudinal axis rotation of the apical vertebra, the vertebral, spinal, and rib cage deformity in idiopathic scoliosis studied by computer tomography. Spine (Phila Pa 1976) 6:567-572, 1981

3. Acaroglu E, Yazici M, Alanay A, Surat A: Three-dimensional evolution of scoliotic curve during instrumentation without fusion in young children. J Pediatr Orthop 22:492-496, 2002

4. Ahmad AA, Aker L, Hanbali Y, Sbaih A, Nazzal Z: Growth modulation and remodeling by means of posterior tethering technique for correction of early-onset scoliosis with thoracolumbar kyphosis. Eur Spine J 26:1748-1755, 2017

5. Akbarnia BA, Breakwell LM, Marks DS, McCarthy RE, Thompson AG, Canale SK, et al: Dual growing rod technique followed for three to eleven years until final fusion: the effect of frequency of lengthening. Spine (Phila Pa 1976) 33:984990, 2008

6. Akbarnia BA, Marks DS, Boachie-Adjei O, Thompson AG, Asher MA: Dual growing rod technique for the treatment of progressive early-onset scoliosis: a multicenter study. Spine (Phila Pa 1976) 30 (17 Suppl):S46-S57, 2005

7. Beguiristain JL, De Salis J, Oriaifo A, Cañadell J: Experimental scoliosis by epiphysiodesis in pigs. Int Orthop 3:317321,1980

8. Cahill PJ, Marvil S, Cuddihy L, Schutt C, Idema J, Clements $\mathrm{DH}$, et al: Autofusion in the immature spine treated with growing rods. Spine (Phila Pa 1976) 35:E1199-E1203, 2010

9. Chen Z, Qiu Y, Zhu Z, Li S, Chen X, Sun X: How does hyperkyphotic early-onset scoliosis respond to growing rod treatment? J Pediatr Orthop 37:e593-e598, 2017

10. Cheung JPY, Yiu KKL, Samartzis D, Kwan K, Tan BB, Cheung KMC: Rod lengthening with the magnetically controlled growing rod: factors influencing rod slippage and reduced gains during distractions. Spine (Phila Pa 1976) 43:E399-E405, 2018

11. Enercan M, Kahraman S, Erturer E, Ozturk C, Hamzaoglu A: Apical and intermediate anchors without fusion improve Cobb angle and thoracic kyphosis in early-onset scoliosis. Clin Orthop Relat Res 472:3902-3908, 2014

12. Fisk JR, Peterson HA, Laughlin R, Lutz R: Spontaneous fusion in scoliosis after instrumentation without arthrodesis. J Pediatr Orthop 15:182-186, 1995

13. Flynn JM, Tomlinson LA, Pawelek J, Thompson GH, McCarthy R, Akbarnia BA: Growing-rod graduates: lessons learned from ninety-nine patients who completed lengthening. J Bone Joint Surg Am 95:1745-1750, 2013

14. Gregersen GG, Lucas DB: An in vivo study of the axial rotation of the human thoracolumbar spine. J Bone Joint Surg Am 49:247-262, 1967

15. Hill G, Nagaraja S, Akbarnia BA, Pawelek J, Sponseller P, Sturm P, et al: Retrieval and clinical analysis of distractionbased dual growing rod constructs for early-onset scoliosis. Spine J 17:1506-1518, 2017

16. Jain A, Sponseller PD, Flynn JM, Shah SA, Thompson GH, Emans JB, et al: Avoidance of "final" surgical fusion after growing-rod treatment for early-onset scoliosis. J Bone Joint Surg Am 98:1073-1078, 2016

17. Kamaci S, Demirkiran G, Ismayilov V, Olgun ZD, Yazici M: The effect of dual growing rod instrumentation on the apical vertebral rotation in early-onset idiopathic scoliosis. J Pediatr Orthop 34:607-612, 2014

18. Kesling KL, Lonstein JE, Denis F, Perra JH, Schwender JD, Transfeldt EE, et al: The crankshaft phenomenon after posterior spinal arthrodesis for congenital scoliosis: a review of 54 patients. Spine (Phila Pa 1976) 28:267-271, 2003

19. Kuklo TR, Potter BK, Lenke LG: Vertebral rotation and thoracic torsion in adolescent idiopathic scoliosis: what is the best radiographic correlate? J Spinal Disord Tech 18:139147,2005

20. Little JP, Izatt MT, Adam CJ, Lofgren O, Sundberg A, Labrom RD, et al: Evaluating the change in axial vertebral rotation following thoracoscopic anterior scoliosis surgery using low-dose computed tomography. Spine Deform 5:172180,2017 
21. McCarthy RE, Luhmann S, Lenke L, McCullough FL: The Shilla growth guidance technique for early-onset spinal deformities at 2-year follow-up: a preliminary report. J Pediatr Orthop 34:1-7, 2014

22. Sankar WN, Skaggs DL, Yazici M, Johnston CE II, Shah SA, Javidan P, et al: Lengthening of dual growing rods and the law of diminishing returns. Spine (Phila Pa 1976) 36:806809,2011

23. Scott JC, Morgan TH: The natural history and prognosis of infantile idiopathic scoliosis. J Bone Joint Surg Br 37B:400-413, 1955

24. Skov ST, Wijdicks SPJ, Bunger C, Castelein RM, Li H, Kruyt MC: Treatment of early-onset scoliosis with a hybrid of a concave magnetic driver (MCGR) and a contralateral passive sliding rod construct with apical control: preliminary report on 17 cases. Spine J 18:122-129, 2018

25. Terek RM, Wehner J, Lubicky JP: Crankshaft phenomenon in congenital scoliosis: a preliminary report. J Pediatr Orthop 11:527-532, 1991

26. Thompson GH, Akbarnia BA, Kostial P, Poe-Kochert C, Armstrong DG, Roh J, et al: Comparison of single and dual growing rod techniques followed through definitive surgery: a preliminary study. Spine (Phila Pa 1976) 30:2039-2044, 2005

27. Urbanski W, Wolanczyk MJ, Jurasz W, Kulej M, Morasiewicz P, Dragan SL, et al: The impact of direct vertebral rotation (DVR) on radiographic outcome in surgical correction of idiopathic scoliosis. Arch Orthop Trauma Surg 137:879885,2017

28. Wang S, Zhang J, Qiu G, Wang Y, Li S, Zhao Y, et al: Dual growing rods technique for congenital scoliosis: more than 2 years outcomes: preliminary results of a single center. Spine (Phila Pa 1976) 37:E1639-E1644, 2012

29. Wang S, Zhang J, Qiu G, Wang Y, Weng X, Guo J: One-stage posterior osteotomy with short segmental fusion and dual growing rod technique for severe rigid congenital scoliosis: the preliminary clinical outcomes of a hybrid technique. Spine (Phila Pa 1976) 39:E294-E299, 2014

30. Zhou X, Zhang H, Sucato DJ, Johnston CE: Effect of dual screws across the vertebral neurocentral synchondrosis on spinal canal development in an immature spine: a porcine model. J Bone Joint Surg Am 96:e146, 2014

\section{Disclosures}

The authors report no conflict of interest concerning the materials or methods used in this study or the findings specified in this paper.

\section{Author Contributions}

Conception and design: Sun, Xu, Qiu. Acquisition of data: Xu, Z Chen, Shi, X Chen. Analysis and interpretation of data: Xu, Shi, Li, Du. Drafting the article: Xu. Critically revising the article: Sun, Qiu, Zhu. Reviewed submitted version of manuscript: Sun, Qiu. Approved the final version of the manuscript on behalf of all authors: Sun. Statistical analysis: Z Chen, Shi. Administrative/ technical/material support: Z Chen, X Chen. Study supervision: Sun, Qiu, Zhu.

\section{Correspondence}

Xu Sun: Nanjing University Medical School, Nanjing, China. drsunxu@163.com. 